Annals of Pure and Applied Mathematics

Vol. 14, No. 1, 2017, 183-188

ISSN: 2279-087X (P), 2279-0888(online)

Published on 9 August 2017

Annals

www.researchmathsci.org

DOI: http://dx.doi.org/10.22457/apam.v14n1a21

Pure and Applied

Mathematics

\title{
Ninth Degree B-spline Collocation Method for Numerical Solution of the Ninth Order Linear Differential Equation
}

\author{
Y.Rajashekhar Reddy
}

Department of mathematics, College of Engineering Jagitial, Nachupally (Kondagattu), Jawaharlal Nehru Technological University, India

Email: yrsreddy4@gmail.com

Received 17 July 2017; accepted 1 August 2017

Abstract. In this paper, collocation method using recursive form of Ninth degree B-spline functions as basis is developed and employed to find the numerical solution for ninth order boundary value problems. Numerical examples are considered to test the performance, stability and accuracy of the present developed method.

Keywords: B-Spline, collocation, recursive, linear differential equation

AMS Mathematics Subject Classification (2010): 65Cxx

1. Introduction

This paper is concerned with the numerical solution of ninth order linear boundary value problem by using ninth degree B-spline collocation solution.

The ninth order linear differential equation with boundary conditions is given as

$$
\begin{gathered}
P O(x) \frac{d^{9} U}{d x^{9}}+P_{1}(x) \frac{d^{8} U}{d x^{8}}+P_{2}(x) \frac{d^{7} U}{d x^{7}}+P_{3}(x) \frac{d^{6} U}{d x^{6}}+P_{4}(x) \frac{d^{5} U}{d x^{5}}+P_{5}(x) \frac{d^{4} U}{d x^{4}}+P_{6}(x) \frac{d^{3} U}{d x^{3}}+P_{7}(x) \frac{d^{2} U}{d x^{2}} \\
+P_{8}(x) \frac{d U}{d x}+P_{9}(x) U=Q(x) x \in(a, b),
\end{gathered}
$$

with the boundary conditions

$U(a)=d_{1}, U(b)=d_{2} \quad U^{\prime}(a)=d_{3}, U^{\prime}(b)=d_{4}, U^{\prime \prime}(a)=d_{5}, U^{\prime \prime}(b)=d_{6}$,

$U^{\prime \prime \prime}(a)=d_{7}, U^{\prime \prime \prime}(b)=d_{8}, U^{i v}(b)=d_{9}$

where $a, b, d_{1}, d_{2}, d_{3}, d_{4}, d_{5}, d_{6}, d_{7}, d_{8}, d_{9}$ are constants.

$P_{0}(x), P_{1}(x), P_{2}(x), P_{3}(x), P_{4}(x), P_{5}(x), P_{6}(x), P_{7}(x), P_{8}(x), P_{9}(x), Q(x)$ are function of $x$.

Different methods have been developed by many authors to solve the ninth order boundary value problems. Homotopy perturbation method was applied to obtain the solution for ninth and tenth order boundary value problems by Tauseef and Ahmet [1]. Samir presented spectral collocation method to solve ninth order boundary value problems [2]. 


\section{Y.Rajashekhar Reddy}

In this paper, recursive form of ninth degree B-spline is employed as basis function in collocation method to solve the ninth order boundary value problems of the type (1)-(2).

\section{Description of method}

The solution domain $\mathrm{a} \leq \mathrm{x} \leq \mathrm{b}$ is partitioned into a mesh of uniform length $\mathrm{h}=\mathrm{x}_{\mathrm{j}+1}-\mathrm{x}_{\mathrm{j}}$, where $\mathrm{j}=0,1,2, \ldots, \mathrm{N}-1, \mathrm{~N}$. Such that $\mathrm{a}=\mathrm{x}_{0}<\mathrm{x}_{1}<\mathrm{x}_{2} \ldots \ldots<\mathrm{x}_{\mathrm{n}-1}<\mathrm{x}_{\mathrm{n}}=\mathrm{b}$.

In the ninth degree $\mathrm{B}$-spline collocation method the approximate solution is written as the linear combination of ninth degree B-spline basis functions for the approximation space under consideration. The proposed numerical solution for solving Eq. (1) using the collocation method with ninth degree B-spline is to find an approximation solution $U^{h}(x)$ to the exact solution $U(x)$ in the form:

$$
U^{h}(x)=\sum_{i=-8}^{n+8} C_{i} N_{i, p}(x)
$$

where $C_{i}$ 's are constants to be determined from the boundary conditions and collocation from the differential equation.

A zero degree and other than zero degree B-spline basis functions $[3,4]$ are defined at $x_{i}$ recursively over the knot vector space

$$
X=\left\{x_{1}, x_{2}, x_{3} \ldots \ldots \ldots x_{n-1}, x_{n}\right\} \text { as }
$$

i) if $p=0$

$$
\begin{array}{lll}
N_{i, p}(x)=1 & \text { if } & x \in\left(x_{i}, x_{i+i}\right) \\
N_{i, p}(x)=0 & \text { if } & x \notin\left(x_{i}, x_{i+i}\right) \\
\text { ii) if } p \geq 1 & &
\end{array}
$$

$$
N_{i, p}(x)=\frac{x-x_{i}}{x_{i+p}-x_{i}} N_{i, p-1}(x)+\frac{x_{i+p+1}-x}{x_{i+p+1}-x_{i+1}} N_{i+1, p-1}(x)
$$

where $\mathrm{p}$ is the degree of the $\mathrm{B}$-spline basis function and $x$ is the parameter belongs to $X$.When evaluating these functions, ratios of the form $0 / 0$ are defined as zero.

\section{Derivation of B-splines}

If $\mathrm{p}=9$

we have

$$
\begin{aligned}
& \dot{N}_{i, p}(x)=\frac{x-x_{i}}{x_{i+p}-x_{i}} N_{i, p-1}^{i}(x)+\frac{N_{i, p-1}(x)}{x_{i+p}-x_{i}}+\frac{x_{i+p+1}-x}{x_{i+p+1}-x_{i+1}} N_{i+1, p-1}(x)-\frac{N_{i+1, p-1}(x)}{x_{i+p+1}-x_{i+1}} \\
& N_{i, p}^{i x}(x)=9 \frac{N^{v i i i}{ }_{i, p-1}(x)}{x_{i+p}-x_{i}}-9 \frac{N^{v i i i} i_{i+1, p-1}(x)}{x_{i+p+1}-x_{i+1}}
\end{aligned}
$$


Ninth Degree B-spline Collocation Method for Numerical Solution of the Ninth Order Linear Differential Equation

$$
\left(U^{h}\right)^{i x}(x)=\sum_{i=-9}^{n+9} C_{i} N^{i x} i, p(x)
$$

The $x_{i}{ }^{\prime} s$ are known as nodes, the nodes are treated as knots in collocation Bspline method where the B-spline basis functions are defined and these nodes are used to make the residue equal to zero to determine unknowns $C_{i}$ 's in (3). Nine extra knots are taken into consideration besides the domain of problem to maintain the partition of unity when evaluating the ninth degree B-spline basis functions at the nodes which are within the considered domain.

Substituting the equations (3) to (6) in equation (1) for $\mathrm{U}(\mathrm{x})$ and derivatives of $\mathrm{U}(\mathrm{x})$. Then system of $(\mathrm{n}+1)$ linear equations are obtained in $(n+9)$ constants. Applying the boundary conditions to equation (2), eight more equations are generated in constants. Finally, we have $(n+10)$ equations in $(n+10)$ constants.

Solving the system of equations for constants and substituting these constants in equation (3) then assumed solution becomes the known approximation solution for equation (1) at corresponding the collocation points.

This is implemented using the Matlab programming.

\section{Numerical scheme}

Example 1. A ninth order differential equation with boundary conditions [5] is considered to test the performance of the proposed method.

$$
\frac{d^{9} y}{d x^{9}}-y=-9 e^{x}
$$

with the boundary conditions $y(0)=1 \quad y(1)=0 \quad y^{\prime}(0)=0 \quad y^{\prime}(1)=-e$

$$
y^{\prime \prime}(0)=-1 y^{\prime \prime}(1)=-2 e y^{\prime \prime}(0)=-2 y^{\prime \prime \prime}(1)=-3 e y^{i v}(1)=-3
$$

The exact solution for the example is $y=x(1-x) e^{x}$

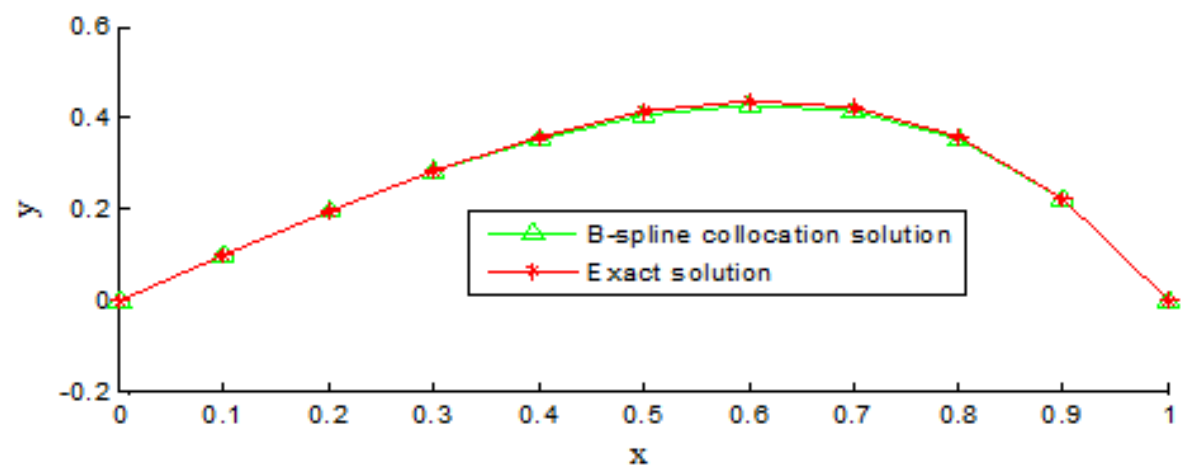

Figure 1: Comparison of ninth degree B-spline collocation solution with exact solution for 11 collocation points 
Y.Rajashekhar Reddy

\begin{tabular}{|l|l|l|l|l|l|l|l|l|l|l|}
\hline Nodes & 0 & .1 & .2 & .3 & .4 & .5 & .6 & .7 & .8 & .9 \\
\hline $\begin{array}{l}\text { Absolute } \\
\text { relative } \\
\text { errors }\end{array}$ & 0 & 0.00 & .0010 & .0043 & 0.0097 & 0.0156 & 0.0194 & 0.0186 & 0.0130 & 0.0048 \\
\hline
\end{tabular}

Table 1: Absolute relative errors at nodes

The obtained numerical solution is compared with the exact solution which is shown graphically in Figure 1. Absolute relative errors are evaluated at different node points and presented in Table 1.

Example 2. Let us consider the following equation.

$$
\begin{aligned}
& \frac{d^{9} y}{d x^{9}}+\frac{d^{7} y}{d x^{7}}+x \frac{d^{4} y}{d x^{4}}+\frac{d^{3} y}{d x^{3}}+\sin x \frac{d y}{d x}+y \\
& =5 x \sin x-\cos x+x^{2} \cos x-x \sin ^{2} x+\sin x \cos x+x \cos x
\end{aligned}
$$

with the boundary conditions $y(0)=0 \quad y(1)=\cos 1 \quad y^{\prime}(0)=1$

$y^{\prime}(1)=\cos 1-\sin 1 \quad y^{\prime \prime}(0)=0 \quad y^{\prime \prime}(1)=-2 \sin 1-\cos 1 \quad y^{\prime \prime \prime}(0)=-3$

$y^{\prime \prime \prime}(1)=-3 \cos 1+\sin 1 \quad y^{i v}(1)=0$

The exact solution for example 2 is given as $y=x \cos x$.

\begin{tabular}{|l|l|l|l|l|l|l|l|l|l|l|}
\hline$x$ & 0 & .1 & .2 & .3 & .4 & .5 & .6 & .7 & .8 & .9 \\
\hline $\begin{array}{l}\text { Ninth degree } \\
\begin{array}{l}\text { B-spline } \\
\text { collocation } \\
\text { solution }\end{array}\end{array}$ & 0.000 & .0995 & .1960 & .2866 & .3684 & .4387 & .4952 & .5354 & .5574 & .5594 \\
\hline Exact solution & 0.000 & .0995 & .1960 & .2866 & .3684 & .4388 & .4952 & .5354 & .5574 & .5594 \\
\hline
\end{tabular}

Table 2: Comparison of B-spline collocation solution with exact solution

Clearly from Table 1 we have seen that the B-spline collocation solution values are meeting with the exact values accurately. Absolute relative errors are presented graphically in Figure 2. 
Ninth Degree B-spline Collocation Method for Numerical Solution of the Ninth Order Linear Differential Equation

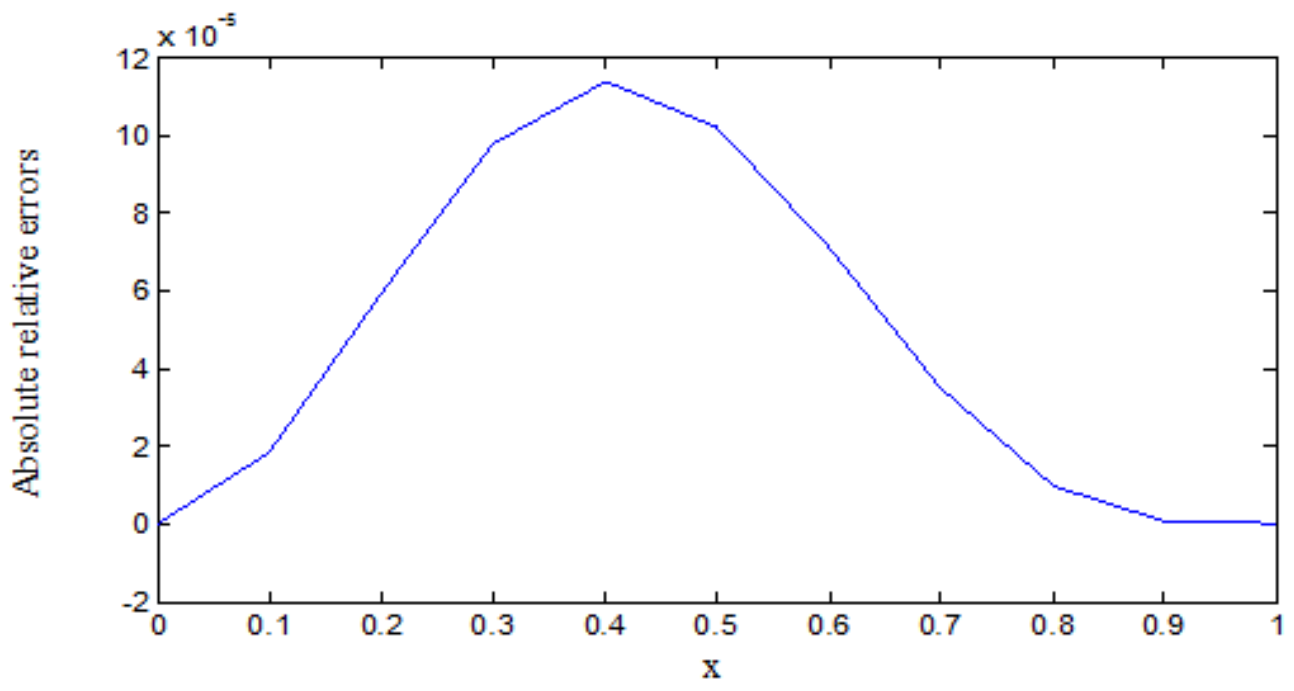

Figure 2:

\section{Conclusions}

In this article, developed collocation method by using the ninth degree B-spline as basis function in collocation method is applied to ninth order linear differential equations with boundary condition problems. It is observed that obtained values are very close to the exact values and further absolute relative errors are very less at the nodes. This shows that the proposed method is effective and useful to find the numerical solutions for ninth order linear differential equation with boundary value problems.

\section{REFERENCES}

1. S.T.Mohyud-Din and A.Yilidirim, Solution of tenth and ninth order boundary value problems by homotopy-perturbation method, J.KSIAM, 14(1) (2010) 17-27.

2. S.K.Bhowmik, Tchebychev polynomial approximations for $m$ th order boundary value problems, Int. J. of Pure and Applied Mathematics, 98(1) (2015) 45-63.

3. H.B.Curry and I.J.Schoenberg, On polya frequency functions IV: The fundamental spline functions and their limits, J. Anal. Math., 17 (1966) 71-107.

4. C.D.Boor, On calculating with B-splines, Journal of Approximation Theory, 62 (1972) 124-135.

5. S.M.Reddy, Collocation method for ninth order boundary problems by using sextic B-splines, Int. Research Journal of Engineering and Technology, 3 (2016) 781-785.

6. Y.Rajashekhar Reddy, Efficient numerical solution for seventh order differential equation by using septic B-spline collocation method with non-uniform length, Int. J. of Pure and Applied Mathematics, 13(7) (2017) 3543-3548.

7. U.Sarder, M.M.Haque and T.Ferdaus, Finite difference solution of mixed convective heat transfer transient flow along a continuously moving cooled plate, Annals of Pure and Applied Mathematics, 7(2) (2014) 18-26. 
Y.Rajashekhar Reddy

8. M.R.Kumar and G.Uthra, A study on numerical stability of finite difference formulae for numerical differentiation and integration, Annals of Pure and Applied Mathematics, 8(2) (2014) 27-36. 